\title{
An estimation of the clinical mastitis incidence per 100 cows per year based on routinely collected herd data
}

\author{
I. M. G. A. Santman-Berends, ${ }^{* 1}$ T. J. G. M. Lam, ${ }^{\star} \dagger$ J. Keurentjes, ${ }^{*}$ and G. van Schaik ${ }^{*}$ \\ *GD Animal Health, PO Box 9, 7400 AA Deventer, the Netherlands \\ †Department of Farm Animal Health, Faculty of Veterinary Medicine, Utrecht University, PO Box 80151, 3508 TD Utrecht, the Netherlands
}

\begin{abstract}
The aim of this study was to evaluate whether it was possible to (1) estimate the clinical mastitis incidence rate (CMI) for all Dutch dairy herds and (2) to detect farms with a high CMI based on routinely collected herd data. For this study, 240 dairy farms with a conventional milking system that participated in the milk recording program every 4 to 6 wk were randomly selected and agreed to participate. From the initial 240 herds, data of clinical mastitis (CM) registrations and routinely collected herd data of 227 herds were complete and could be used for analysis. Routinely collected herd data consisted of identification and registration records, antimicrobial usage, test-day records from the milk recording program, bulk tank milk (BTM) somatic cell count data and results of diagnostic tests on BTM samples. For each of the 227 herds, the CMI per 100 cows per year was calculated per quarter of the year and was combined with the available herd data. Two models were developed to predict the CMI for all dairy herds and to detect individual herds that belonged to the $25 \%$ herds with the highest CMI. Records of 156 $(67 \%)$ herds were used for development of the models and the remaining $71(33 \%)$ were used for validation. The model that estimated the CMI in all herds consisted of 11 explanatory variables. The observed and predicted averages of the validation herds were not significantly different. The model estimated a CMI per 100 cows per year of 32.5 cases $(95 \%$ confidence interval $=30.2-34.8)$, whereas the farmers registered 33.4 cases $(95 \%$ confidence interval $=29.5-37.4)$. The model that aimed at detecting individual herds with a high CMI contained 6 explanatory variables and could correctly classify $77 \%$ of all validation herds at the quarter-year level. The most important variables in the model were antibiotic usage for treating CM and BTM somatic cell count. In conclusion, models based on routinely collected herd data gave an accurate prediction of CMI
\end{abstract}

Received March 28, 2015.

Accepted June 29, 2015.

${ }^{1}$ Corresponding author: i.santman@gdanimalhealth.com for all Dutch dairy herds and could detect individual dairy herds with a high CMI. With these models it is possible to periodically monitor CMI both at the herd and at the national level, which is valuable for monitoring purposes and can motivate farmers to continuously improve udder health in their herds.

Key words: dairy cattle, clinical mastitis, predictive modeling, routinely collected herd data

\section{INTRODUCTION}

Clinical mastitis (CM) is a frequently occurring, economically important disease for dairy industries around the world (Olde Riekerink et al., 2008; Lam et al., 2013). Udder health is often monitored based on individual or bulk tank milk (BTM) SCC data. In some European countries, CM records are registered in disease-recording systems. However, these registrations underestimate the CM rates by 20 to $100 \%$ (Wolff et al., 2012). In the Netherlands, the clinical mastitis incidence (CMI) is an important indicator for both animal health and welfare, which is not uniformly registered on a routine basis for all dairy herds and, therefore, cannot easily be monitored. Monitoring CMI provides insight in the trend in time and enables early detection of unfavorable alterations. On the herd level, monitoring CMI provides farmers more insight in their own situation compared with other farms, which may motivate them to improve udder health. A decrease in CMI will have a positive effect on animal health, animal welfare, antimicrobial use (AMU), work pleasure, and net return of the farm (Huijps et al., 2008; Jansen et al., 2010; Trevisi et al., 2014).

In the Netherlands, the most recent CMI estimation was in 2009 and showed a slight decrease over the period January 1, 2005, and December 3, 2009, from 33.5 to 28.1 cases/100 cows per year, respectively (Lam et al., 2013). However, for these estimates of CMI farmers had to observe, register and communicate all cases of $\mathrm{CM}$ in the herd for a year, which is very labor intensive. Therefore, a need exists for an alternative method to estimate and monitor CMI. In previous studies, it has been shown that elevated SCC is indicative for IMI and 
often results in CM (Berning and Shook, 1992; de Haas et al., 2005; van den Borne et al., 2010). Nevertheless, not all elevated SCC cases result in CM. In addition, in routinely collected data, such as BTM records and testday milk recording, SCC records of cows with CM are generally excluded. Whether routine herd data has the ability to predict CMI on herd and national level has not previously been studied. Currently, besides SCC records, other routine herd data possibly associated with $\mathrm{CM}$, such as AMU for intramammary and dry cow treatment, grazing management, milk production, herd size, animal movements, age, and parity, are also available.

The aim of the current study was to evaluate the possibility to use routine herd data to estimate the CMI on year and quarter-year level for the population of Dutch dairy herds. Further, we investigated the potential of routine herd data for comparing the CMI of individual herds to a benchmark of all dairy herds.

\section{MATERIALS AND METHODS}

\section{Study Population}

For our study, all dairy herds with a conventional milking system that participated in milk recording program on a 4- to 6-wk interval of the Dutch Royal Cattle Syndicate (CRV, Arnhem, the Netherlands) were eligible for inclusion (parameters that were available in the milk recording data are described in Appendix Table A1). Herds with an automatic milking system (AMS) were excluded because of the differences in detection of mastitis compared with herds with a conventional milking system. Farms that did not participate in the milk-recording program were excluded because of the lack of SCC data. Of the total population of 17,459 Dutch dairy herds, 12,490 met the inclusion criteria (18\% of the herds were excluded because of the use of an AMS and $10 \%$ were excluded because they did not participate in the milk-control program). The study population was randomly split in 2 groups of dairy herds: one group for the development and one for the validation of the prediction models (split-validation method; Steyerberg, 2009). With the program Winepiscope 2.0 (sample size to estimate a mean; Thrusfield et al., 2001), we estimated that at least 200 dairy herds had to be included to be able to obtain a precise estimation (accepted error in CMI <6) of the CMI in both subpopulations, assuming that the expected CMI would be 25 to 30 cases per 100 cows per year and the expected standard deviation would be 22 cases per 100 cows per year (Lam et al., 2013). In addition, with at least 70 herds in the validation group, it was possible to detect differences between observed and predicted
CMI of $7.5 \%$ or higher based on the previous mentioned assumptions. Based on previous experience (van den Borne et al., 2010; Lam et al., 2013), the drop-out percentage was expected to vary between 10 and $20 \%$, and therefore 40 additional dairy herds had to be included. Assuming a response percentage of 15 to 20\%, 1,350 randomly selected dairy herds were contacted by mail to participate in our study. The first 240 farmers that responded were included in the study. Participation meant that they were obliged to observe, register, and communicate all CM cases in their herd to GD Animal Health on a monthly basis from January 1 to December 31,2013 . They also gave consent for use of their routine herd data. In return, BTM was tested 10 times during the year on the presence of udder pathogens for free and, when the study had finished, participating herds received an overview of the udder health of their herds compared with the other participants.

\section{Definitions and Collection of Herd Data}

At the start of the study, all 240 farms were visited by an employee of GD Animal Health. During these visits, the aim and the methods of the study were explained. Farmers used a uniform definition of CM and used standard forms to register and report the CM cases. The definition of CM was every abnormality on udder or milk observed by the farmer (Lam et al., 2013). Abnormalities included alteration in color or consistency of the milk, swollen or red quarters, and clinical signs in cows such as depression, anorexia, dehydration, or fever (Lago et al., 2011). Analogous to previous studies evaluating CM in the Netherlands (Barkema et al., 1999; van den Borne et al., 2010, 2011), every CM case diagnosed by the farmer was considered a new case of $\mathrm{CM}$, with the only exception that CM events occurring within $14 \mathrm{~d}$ in the same quarter were assumed to be the same case and were excluded from analysis. For each herd, the CMI was expressed as the number of quarter cases per 100 cows at risk per year and was calculated as the number of quarter cases of CM divided by the number of cow days at risk (DAR) multiplied by $365 \mathrm{~d}$ and 100 cows (equation 1):

$$
\mathrm{CMI}=(\mathrm{CM} / \mathrm{DAR}) \times 100 \times 365 .
$$

Based on the identification and registration data, DAR was calculated as the total sum of the number of days cows were present at the farm during at least $1 \mathrm{~d}$ in the study period, accounting for the moment of entrance and removal in the herd. An exception was made for primiparous cows, who became at risk at their first calving (and were assumed not at risk before this date). Cows were assumed to be always at risk for CM, 
because even if they suffered from CM in a quarter they were still at risk for developing $\mathrm{CM}$ in one of the other quarters.

To minimize response bias, after each month the farmers were asked to send in forms with the identification numbers of cows diagnosed with CM, the quarters in which an abnormality was observed, the date on which CM was observed, and whether or not the cases were treated with antibiotics. Farmers that did not submit the form at the end of the month were contacted by mail around the seventh day of the subsequent month and were contacted by phone every following week. With a program, developed in Stata 13.1 (Stata, 2014), we monitored whether the farms regularly submitted the registration forms twice a week. In addition, this program was also used to detect incompleteness in the submitted forms and data-entry errors. The following abnormalities could occur: (1) the unique herd number was entered with typing errors, (2) there were typing errors in the identification of the cow, or (3) the data that was submitted was incomplete. The first 2 types of errors could be corrected by rechecking the forms. When the submitted data appeared incomplete the farmer was contacted to complete the data.

\section{Routinely Collected Herd Data}

Besides the CM data, other herd data were routinely collected. Identification and registration records that contained all animal registrations and movements were available in the study period from January 1 until December 31, 2013 (provided by the Dutch enterprise agency, RVO, The Hague, the Netherlands). Based on these data, the number of cows and DAR could be calculated for every herd in each quarter of the year (January-March, April-June, July-September, and October-December). In addition, these data were also used to provide insight in growth in herd size, replacement rates, and purchase of cattle. Location of the herd (i.e., province) were provided by GD Animal Health, Deventer, and test-day records from the milk recording program on animal and herd level were provided by CRV. From these data, for each cow, the distinction between primiparae and multiparae was made. In addition, whether a cow suffered from a (new) elevated SCC indicating subclinical mastitis (SCM) was derived on test-day level. In our study the threshold of $>250,000$ cells/mL was used to diagnose SCM in multiparae and a threshold of $>150,000$ cells $/ \mathrm{mL}$ was used in heifers, analogous to the definitions used in the Netherlands (Sampimon et al., 2010; CRV, 2011). On the herd and quarter-year level, the prevalence and incidence of SCM was derived as well as net return (based on 305-d production corrected for fat or protein, age, and calv- ing season), standardized milk production (production level of the herd on a specific test-day; CRV, 2001), raw milk production (in kilograms), proportion primiparae, mean percentage fat, mean percentage protein, mean percentage lactose, and mean percentage urea.

Bulk tank milk SCC records were provided by Qlip laboratories (Zutphen, the Netherlands) and contained the SCC for each of the participating herds on a biweekly level for the complete study period. Data on AMU originated from the Dutch MediRund database (provided by the Dutch dairy association, NZO, The Hague, the Netherlands). This data consisted of all data on antimicrobials delivered by veterinarians to each of the participating farms from January 1 to December 31, 2013. From this data the animal daily dose of antibiotics (ADD) was calculated per herd for each quarter of the year according to the definitions provided by the Dutch Veterinary Medicines Authority (SDA, 2014).

Finally, every month a BTM sample of each participating herd was cultured for coliforms, streptococci, Staphylococcus aureus, Klebsiella spp., Streptococcus agalactiae, Streptococcus dysgalactiae, and Streptococcus uberis. For a thorough description of the parameters that were evaluated in the models, please refer to the Appendix.

\section{Validation of the Data}

From the sum of the number of CM cases divided by DAR and multiplied by $365 \mathrm{~d}$ and 100 cows (equation 1), the CMI for each herd was calculated and the herd-level CMI was combined with the collected routine herd data. Subclinical udder health parameters were calculated on the herd level using test-day records and parity (primiparae/multiparae; Appendix). From the milk-recording program, a total of 223,791 test-day records were available from 28,005 cows between January 1, 2013, and February 15, 2014. The records of the first 6 wk in 2014 were included to evaluate whether elevated SCC quarters recovered after the last test-day in 2013. Parameters describing antibiotic use in cows $\geq 2$ yr old were calculated using the definitions provided by the Dutch Veterinary Medicines Authority and which is described in Santman-Berends et al. (2014). In short, for each herd and quarter-year the total treated weight in kilograms per cow were calculated by multiplying the amount of prescribed of antibiotic product by the amount of active substance, divided by prescribed dose per animal weight in kilograms. Subsequently, the ADD per herd per quarter-year was calculated by dividing the amount of treated weight by a fixed total weight of cows ( $\geq 2$ yr old, $600 \mathrm{~kg} / \mathrm{cow}$ ) present in the herd. In addition to the total ADD, the ADD was also calculated for 
intramammary treatment of CM, dry cow treatment, and parental treatment in milking cows separately. All data were evaluated at quarter-year level.

\section{Predictive Modeling}

The 227 herds of which both the CMI could be calculated and all routinely collected herd data were available were randomly assigned to 1 of 2 subpopulations. The first subpopulation consisted of 156 (67\%) of the participating herds that were used to develop the predictive models (training data set). The remaining 71 $(33 \%)$ herds were used for validation of the developed models (validation data set; Steyerberg, 2009).

Two different prediction models were developed in Stata 13 (Stata, 2014). The first model was a generalized estimating equation (GEE) population average model with a Gaussian distribution, an independent correlation structure, and an identity link function. With this model, the analysis was conducted relative to the population mean to be able to correct for repeated measures within herds. This linear model was not used for estimating the CMI for individual herds because the fit of this model for individual herds was not sufficient (results not shown). Therefore, a second model that aimed at detecting individual herds with a high CMI (belonging to the $25 \%$ herds with the highest CMI or not) was developed. In this model, the dependent variable had a binomial distribution and a GEE population average model with a binomial distribution, an independent correlation structure and a Logit link function was used to predict whether a herd belonged to the $25 \%$ herds with the highest CMI.

In the first model, the CMI that was observed and registered by the farmer on herd and quarter-year level was included as a dependent variable. All parameters (Appendix) from the routine herd data were evaluated in the model. Whether a variable was retained in the model was evaluated based on its added value to the fit and the predictive capabilities of the model. First, univariable analyses were conducted on each of the parameters and parameters with $P<0.25$ were retained for inclusion in the multivariable model. Subsequently, a forward stepwise selection and elimination method was used to obtain the best fitted model by monitoring the quasi-likelihood under the independent model criterion (QIC). The best predictive model was defined using the QIC value, which is a general applicable method to select the best fitting model in GEE analyses (Pan, 2001; Cui, 2007). The subset of parameters in the model that resulted in the lowest QIC value was considered the most optimal model. For the final model, all biologically relevant interactions were evaluated and possible collinearity problems were monitored using the variation inflation factor. Further, during model selection, confounding was monitored by the change in the coefficient of a variable after removing another variable (Dohoo et al., 2009). If the change of the estimates exceeded $25 \%$, the removed variable was considered a potential confounder and was re-entered in the model. Thereafter, the data of the 71 remaining herds was used to validate the ability of the model to predict the CMI. Based on a postestimation linear prediction in Stata (2014), the CMI was predicted for each of the validation herds. Both the observed and predicted CMI were combined for all herds and compared, both on the quarter-year and year level, to evaluate whether the model was capable of accurately predicting the CMI for all dairy herds. The robustness of the developed model was evaluated by conducting 2 additional validation methods: (1) validation on 500 bootstrap data sets each containing observations of 71 herds that were extracted from the group of validation herds and (2) cross-validation by varying the herds in the development (90\% of the herds) and validation groups (10\% of the herds) and repeating this 10 times so that every herd was once in the validation data set.

The second predictive model aimed at detecting herds that belonged to the group of $25 \%$ herds with the highest CMI on the quarter-year level. A herd was assigned such an attention if the CMI per 100 cows per year exceeded 45.4, 39.3, 45.9, or 44.0 in the 1st, 2nd, 3rd, or 4th quarter of 2013, respectively. All routinely available parameters were subjected to univariable analyses and were evaluated as to whether they improved the model fit $(P<0.25)$. In the multivariable model, again a forward stepwise selection and elimination method was used to obtain the best fitting model. This was done by evaluating the area under the curve (AUC) of the receiver operating characteristic curve. The calibration of the risk score was assessed by comparing the model predictors to the observed and registered values. The final model had the highest AUC and the highest positive and negative predictive values. The default cut-off value of 0.5 was used to classify herds as belonging to the $25 \%$ herds with $(\geq 0.5)$ or without $(<0.5)$ a CMI attention on the quarter-year level. The final risk score for a herd was validated based on the positive and negative predictive value and AUC of the observations of the 71 validation herds.

\section{Descriptive Results}

\section{RESULTS}

At the end of 2013, 233 out of the 240 farmers that participated in the study completed $\mathrm{CM}$ registration. Together they registered 7,106 quarter cases of CM from 4,947 different cows. From these, 346 observa- 
tions were excluded because multiple $\mathrm{CM}$ cases within $2 \mathrm{wk}$ in the same quarter were combined as $1 \mathrm{CM}$ case. The final CM data consisted of 6,760 CM cases on the quarter level in 4,947 different cows and 7,966,844 DAR (average $329 \mathrm{~d} /$ cow present) in 233 dairy herds. When combining the data, observations of 3 herds were lost because the farms stopped participating in the milkrecording scheme during the study period and observations of 3 additional herds were excluded because the farmers withdrew their consent to use their data on AMU. The final data set consisted of complete observations of 227 herds.

In total, 26,762 cows in the 227 dairy herds that participated in our study were at risk for developing $\mathrm{CM}$ on at least $1 \mathrm{~d}$ in 2013. Per herd, a median number of 91 cows ( $\geq 2$ yr old; mean 104) were present. The participating herds appeared slightly larger than the Dutch average of 94 cows $\geq 2$ yr old at the end of 2013 (based on the I\&R census data, The Hague, the Netherlands). The participating herds were randomly distributed across the country and were, therefore, representative for the distribution of all Dutch dairy herds.

In the 227 dairy herds, CM cases were observed 6,656 times in 4,874 different milking cows. Farmers decided to treat 4,778 (72\%) CM cases with antibiotics. In $94 \%$ of the cases only 1 quarter of the cow was affected with CM. In $5 \%$ of the cases, 2 quarters were affected; in 0.5 and $0.4 \% 3$ or 4 quarters, respectively, with $\mathrm{CM}$ simultaneously were observed.

In the 227 participating herds, the median CMI was 27.8 cases per 100 cows per year $(25$ th percentile $=16.7$; 75 th percentile $=44.0)$. The mean CMI was slightly higher with 32.1 cases per 100 cows per year because of a small number of farms with an extremely high CMI (Figure 1).

\section{Prediction of CMI for all Dairy Herds Based on Routinely Collected Data}

In total, 39 parameters were available for inclusion in the predictive model (Appendix). After univariable analyses, 22 parameters improved the model, reduced the QIC $(P<0.25)$, and were eligible for evaluation in the multivariable prediction model. Three parameters (i.e., total number of cows present in the herd, overall prevalence of cattle with elevated SCC, and incidence of SCM in multiparae based on the definitions of CRV) were not included in the multivariable model because of high correlation with other predictive parameters ( $\mathrm{r}$ $>0.60)$.

The most optimal model that was developed based on the observations of the training data set of 156 herds explained most variation and contained 11 parameters (Table 1). The residuals of the final model were moni- tored for normality based on the skewness and kurtosis and appeared acceptable, although the kurtosis was fairly high. Therefore, in sensitivity analyses, prediction models were developed on the training data set using either a Poisson or negative binomial distribution with the number of $\mathrm{CM}$ cases as dependent variable and the number of cattle days at risk as offset (results not shown). In both analyses, the most optimal model contained exactly the same predictive parameters as the model using the normal distribution. Thus, only the results of the linear model are presented. The variation inflation factor showed that no multicollinearity problems existed in the final linear model. The developed prediction model explained $35 \%$ of the variation in CMI. The parameters with the highest predictive value were the amount of antibiotics provided for intramammary treatment and the BTM SCC. These 2 parameters accounted for $65 \%$ of the variation that was explained by the final model. The remaining 9 parameters accounted for the remaining $35 \%$ of variation explained by the model.

The model predicted an average CMI of 32.5 (95\% CI $=30.2-34.8)$ per 100 cows per year based on the observations of the 71 validation herds. The CMI observed and registered by the farmers was not significantly different, with a CMI of $33.4(95 \%$ CI $=29.5-37.4)$. The calibration plot showed that the average trend line of the validation herds was very close to the most optimal $45^{\circ}$ line (Figure 2). Nevertheless, the difference between the observed and predicted CMI of individual herds could be considerable, especially with observed CMI in the lowest and highest range (Figure 2). The ability of the model to predict extreme high CMI values was

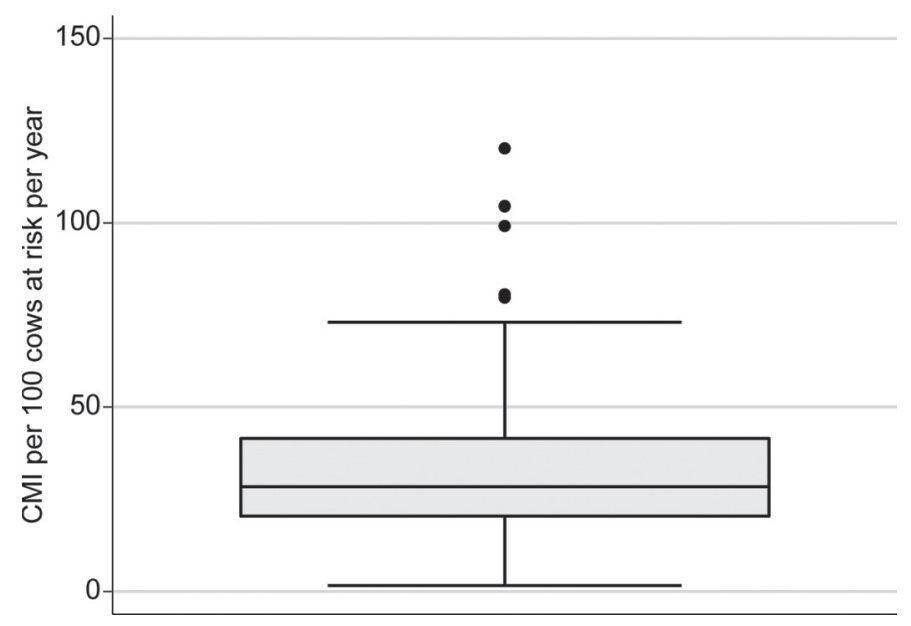

Figure 1. Clinical mastitis incidence (CMI) rate per 100 cows per year in 227 Dutch dairy herds in 2013. The box describes the 25th, 50 th, and 75 th percentiles of the CMI. The whiskers present the 25th percentile -1.5 times the interquartile range and the 75 th percentile +1.5 times the interquartile range. The dots represent the extreme values. 
Table 1. Parameters in the most optimal predictive model for clinical mastitis incidence rate (CMI) per 100 cows at risk per year for each of the 4 quarters in 2013 in 156 Dutch dairy herds (training data set)

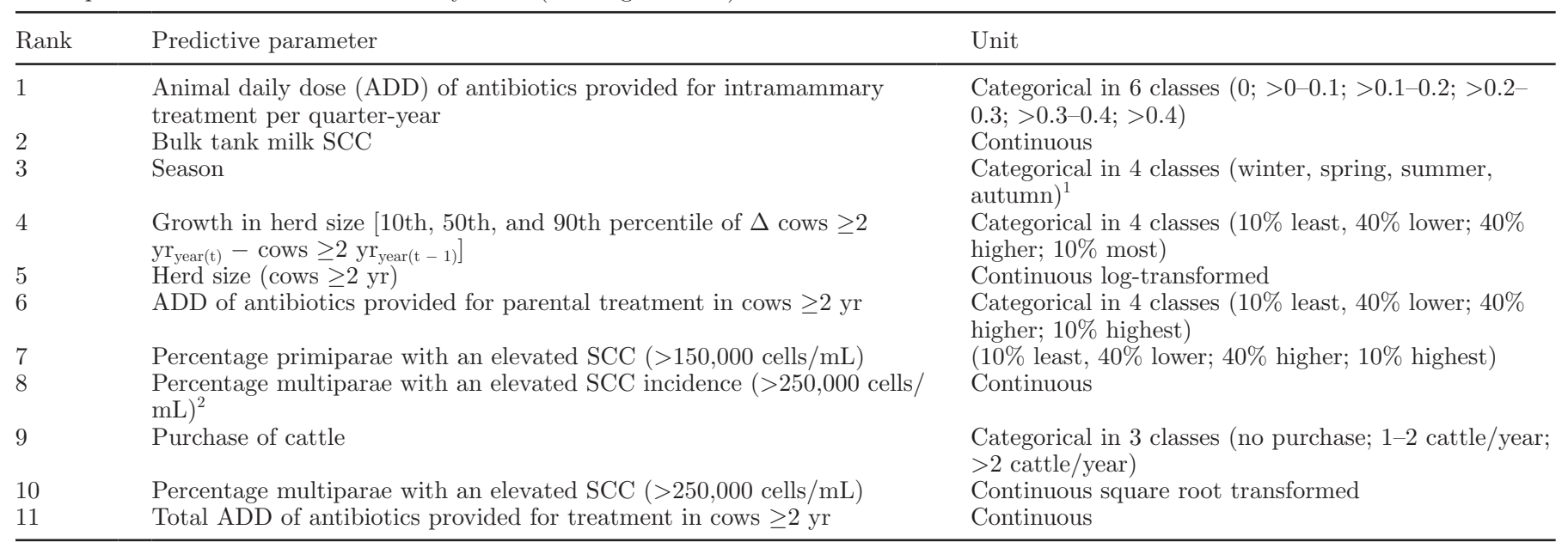

${ }^{1}$ Winter is represented by the first quarter, spring by the second quarter, summer by the third quarter, and autumn by the fourth quarter of the year.

${ }^{2}$ Calculated as the number of cows with a new elevated SCC divided by the number of days at risk as described in the paper of (Santman-Berends et al., 2012).

limited, possibly because some key predictor variables were not available in the data that was assessed, and thus a prediction for CMI for individual herds was not sufficiently valid (Figure 2).

The model was also able to accurately predict the average CMI in the group of validation herds for each quarter-year (Figure 3). In the validation data set, no significant difference was observed in the 4 quarters of 2013 for the predicted and observed CMI (Figure $3)$. The maximum difference between the model predictions and the registered CMI by the farmer was observed in the third quarter (Figure 3). In that quarter, the average prediction was 2.8 cases per 100 cows per year lower than the registered CMI.

The difference between the observed and predicted mean CMI of each of the 500 data sets (that were developed by bootstrapping samples of 70 herds from the 71 herds that were used for validation) varied between a minimum of -5.4 and a maximum of $5.9 \mathrm{CM}$ cases per 100 cows per year. On average, the model slightly underestimated the CMI in the 500 bootstrap data set, at 1.7 cases $(95 \% \mathrm{CI}=1.6-1.9)$ per 100 cows per year. Varying the farms that were assigned to the training and validation data sets did not result in a different model, indicating that the developed model was very robust (results not presented).

\section{Detection of Individual Dairy Herds that Belong to the Group of Herds with the Highest CMI}

The prediction of CMI as a continuous measure for individual herds appeared to not be precise enough. For $45 \%$ of the herds, the predicted CMI differed by
10 or more cases per 100 cows per year compared with the CMI that was observed by the farmer (Figure 2). Therefore, it was decided to attempt to detect herds with the highest CMI. The best model contained 6 parameters and had an AUC of $0.80(95 \% \mathrm{CI}=0.76-0.84)$ in the training data set, using the default cut-off value of 0.5 (Table 2). The amount of prescribed antibiotics for intramammary treatment had the highest predictive value followed by herd size and BTM SCC.

When applying the developed model to the validation data set of 71 dairy herds the AUC was $0.79(95 \%$ $\mathrm{CI}=0.73-0.85)$. The model was able to correctly classify $77.1 \%$ of the observations on the quarter-year level (219 of the 284 observations). Using the default cut-off value of 0.5 , the specificity was high, at $96 \%$, but the sensitivity was $32 \%$. The model could correctly classify 193 observations as no attention herds and 26 observations as attention herds. The negative predictive value of the model was $78 \%$ and the positive predictive value was $72 \%$.

Ten herds were predicted to need attention by the model, whereas this was not supported by the observations and registrations of the farmer. However, in 7 of these herds the registered CMI was only slightly lower than the cut-off value that was used to assign herds as needing attention (Figure 4). These herds had a higher than average percentage of cows with an elevated SCC and were prescribed a higher than average amount of antibiotics for intramammary treatment in the misclassified quarter of 2013.

In 55 of the 284 observations on quarter-year level, the farmers observed and registered their farm as needing attention (a CMI that belonged to the 25\% highest 


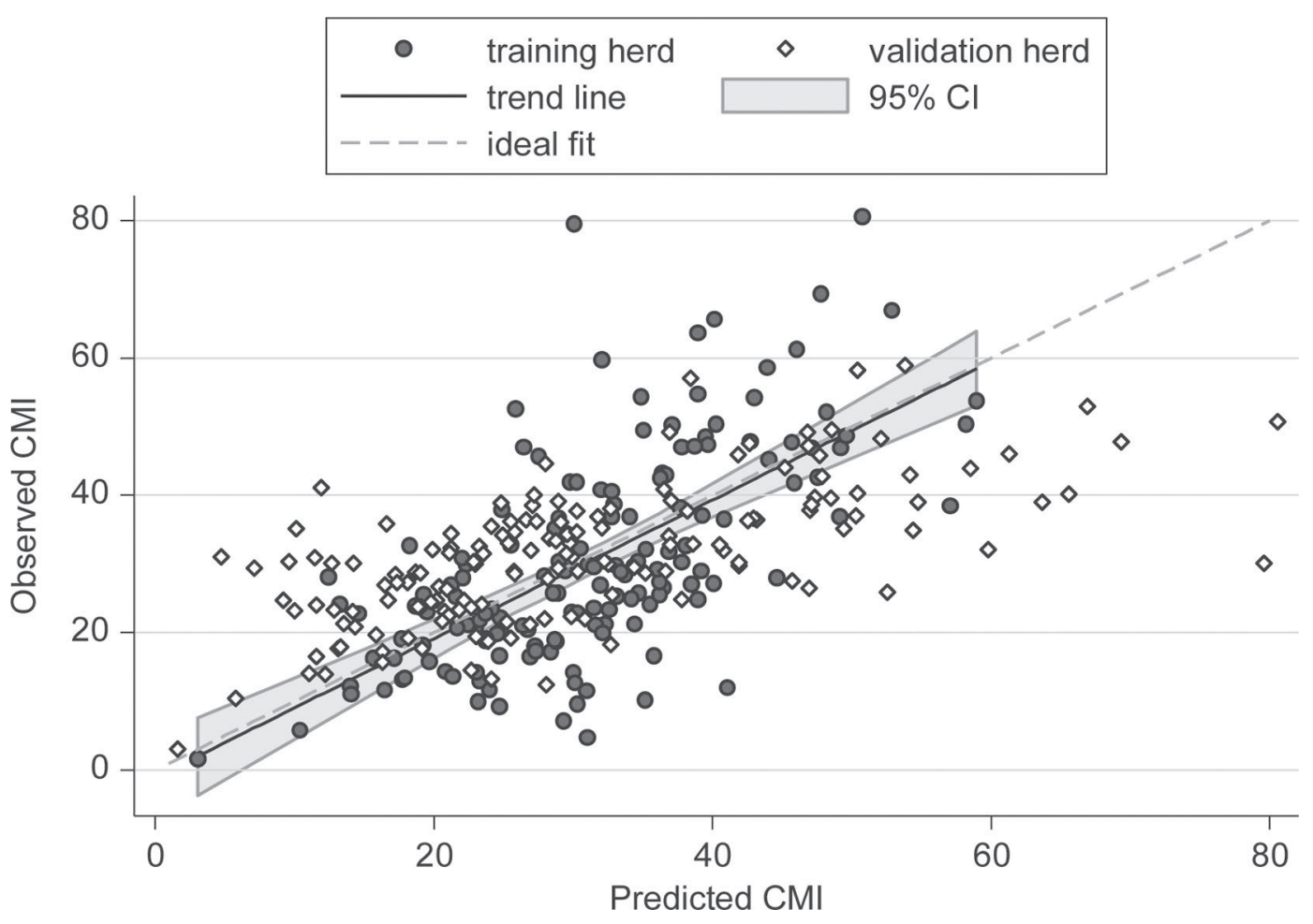

Figure 2. Calibration plot of the observed clinical mastitis incidence (CMI) versus the predicted CMI, containing the observed and predicted CMI of the training $(\mathrm{n}=156)$ and validation herds $(\mathrm{n}=71$ herds) with the trend line of the validation herds (including $95 \%$ CI $)$ and the ideal $45^{\circ}$ line. The CMI of 2 very extreme herds $(\mathrm{CMI}>100)$ that were part of the training data set are not displayed to improve the readability of the figure.

values), whereas this was not supported by the predictions of the model. Almost all herds that received false attention did need attention in one of the other quarters of 2013. There were only 2 herds in which the farmer did not observe a high CMI in any of the quarters of 2013, whereas the model did assign a herd as needing attention in one or more of the quarters of 2013. Most of the observations were close to the cut-off values that were used (Figure 4). The herds in which false attention occurred appeared to have a lower than average percentage of cows with an elevated SCC, had a lower than average BTM SCC, and appeared to treat a relatively low percentage of observed CM cases with antimicrobials $(61 \%$ of $\mathrm{CM}$ cases treated with antibiotics versus $72 \%$ in the study herds).

\section{DISCUSSION}

This paper presents an attempt to estimate the CMI for Dutch dairy herds in 2013. The method used showed potential to predict the average CMI for all dairy herds based on routine herd data. Further, a second model was able to correctly predict $77 \%$ of the individual herds on quarter-year observations as being at risk for having a high CMI (i.e., belonging to the $25 \%$ dairy herds with the highest CMI).
To our knowledge, this is the first time an attempt has been made to predict CMI on routinely collected data. Nevertheless, prediction models are commonly used in human medicine (Lemeshow et al., 1993) and genetic modeling (McGill et al., 2014; Sun et al., 2014). Currently, in many countries, herd data are routinely

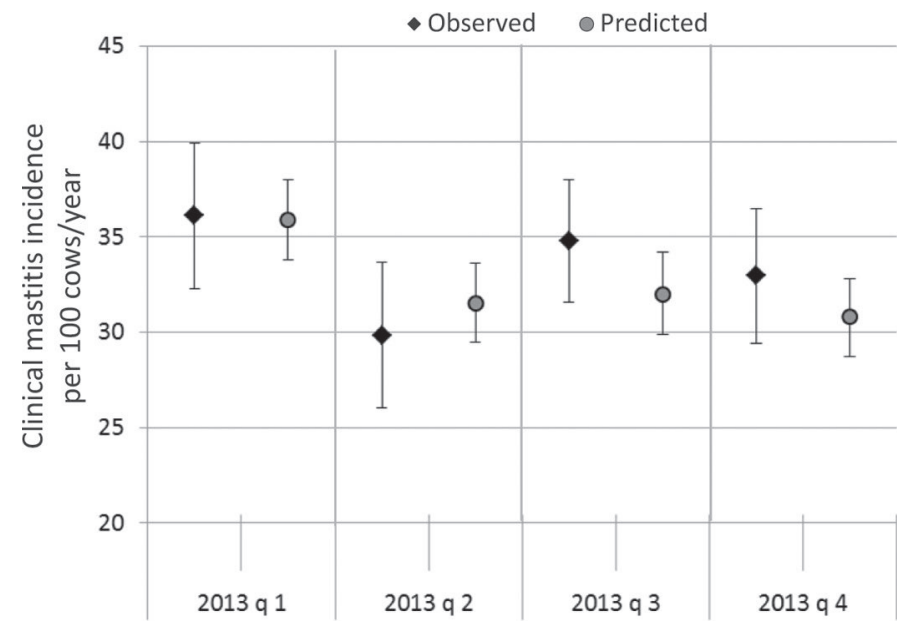

Figure 3. Mean and 95\% CI of the observed and predicted mean clinical mastitis incidence rate (CMI) per 100 cows per year for the validation data set of 71 Dutch dairy herds, respectively, for each quarter of 2013 . 
Table 2. Parameters in the best predictive model for herds belonging to the $25 \%$ of dairy herds with the highest CMI per quarter in 2013 based on CMI observations of 156 farms per quarter-year (training data set)

\begin{tabular}{lll}
\hline Rank & Predictive parameter & Unit \\
\hline 1 & Animal daily dose of antibiotics provided for intramammary & Categorical in 6 classes $(0 ;>0-0.1 ;>0.1-0.2 ;>0.2-0.3 ;$ \\
& treatment per quarter-year & $>0.3-0.4 ;>0.4)$ \\
2 & Herd size (cows $\geq 2$ yr) & Continuous log-transformed \\
3 & Bulk tank milk SCC & Continuous \\
4 & Season & (Categorical in 4 classes: winter, spring, summer, autumn) \\
5 & Standardized milk production & Continuous \\
6 & Percentage primiparae with an elevated SCC incidence & (10\% least, $40 \%$ lower; $40 \%$ higher; $10 \%$ highest $)$ \\
\hline
\end{tabular}

${ }^{1}$ Winter is represented by the first quarter, spring by the second quarter, summer by the third quarter, and autumn by the fourth quarter of the year.

collected because of cattle improvement schemes, milk quality control, regulations, and surveillance, although the exact mix of data may differ. Collection of routinely collected data presents increasing opportunities to apply predictive modeling in veterinary medicine (de Vries et al., 2014; McParland et al., 2014; Vergara et al., 2014). The predictive model for the average CMI
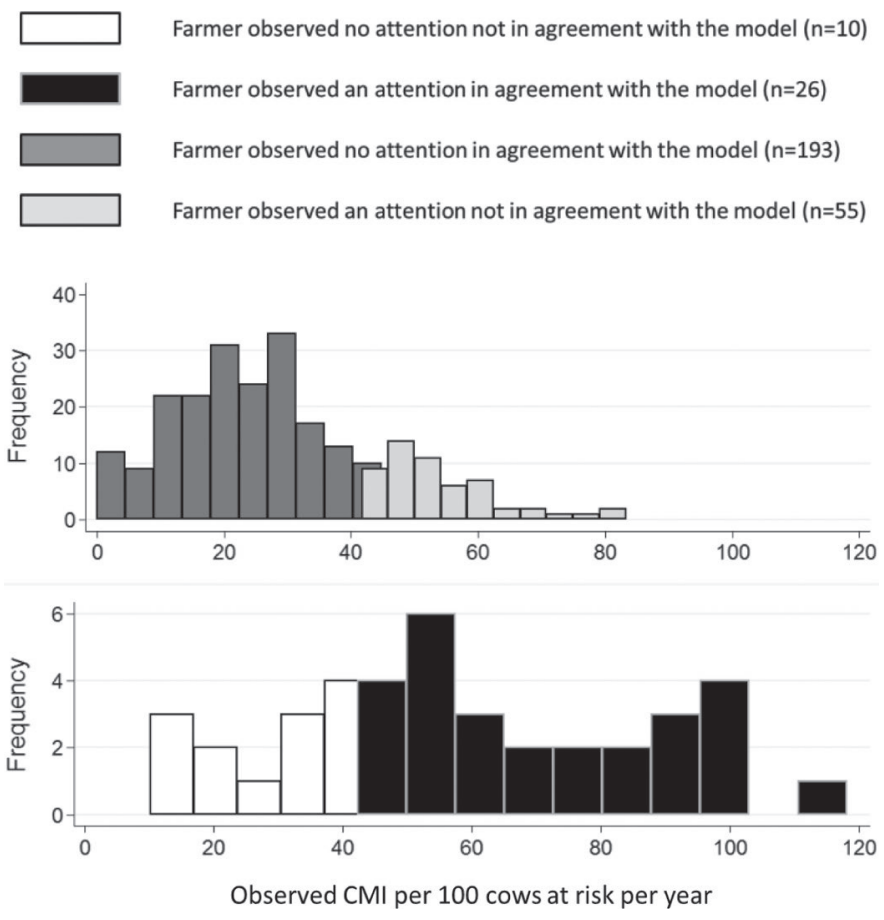

Figure 4. Histograms of the predicted versus observed attentions for the observed clinical mastitis incidence (CMI) per 100 cows per year for each of the 71 validation herds per quarter of 2013 . The cut-off values classified as needs attention (i.e., belonging to the $25 \%$ herds with the highest CMI) were calculated for each quarter separately at $45.4,39.3,45.9$, or 44.0 in the 1 st, 2 nd, 3rd, or 4th quarter of 2013 , respectively. A herd needing no attention was defined as belonging to the $75 \%$ herds with the lowest CMI either registered by the farmer (observed) or predicted by the model (predicted). Because of the different cut-off values used in each quarter of the year, an overlap in the different groups was seen (i.e., a CMI of 42 if classified as an attention in the second quarter whereas it is classified as no attention in the first, third, and fourth quarter of the year). of all dairy herds accurately estimated the CMI and was not significantly different from the average CMI that was observed and registered by the farmers. The residuals of the final model were checked for normality and were classified as acceptable. Results of the linear model were robust given that log-linear and logit models gave the same results. Because the linear model met the aim of the study best (to estimate the average CMI for all dairy herds), it was decided to use a generalized linear model instead of Poisson or negative binomial regression models. The linear model had its limitations in correctly predicting extreme values of CMI, especially underestimating the high CMI values. This was visible in the limited variation that was explained by the model and was also visible in the results of the model predictions for the validation data set and for the 500 bootstrap data set that were used for validating the results. The predicted CMI slightly underestimated the observed CMI in both cases. Nevertheless, because herds with an extreme high CMI are rare, the underestimation of the average CMI for all herds was only limited. Because the predicted and observed CMI for individual herds differed more than 10 cases in $45 \%$ of the predictions, it was decided that the model was not suitable for estimation of the CMI as a continuous measure for individual herds. Instead a model that aimed at detecting farms belonging to the $25 \%$ herds with the highest CMI was developed. This model correctly classified $77 \%$ of the validation herds. Nevertheless, the model still misclassified $23 \%$ of the observations. Studying the misclassified herds more closely, we learned that 2 herds were assigned as needs attention in multiple quarters of 2013, whereas the farmer always observed a low CMI. Conversely, in 11 herds the model did not assign one of the quarters of 2013 as needing attention, whereas the farmers observed high CMI in at least 2 quarters. Misclassification by the model may be questionable in these cases. For the model, the observations of the farmers were used as reference; however, some farmers might have under- or overestimated the amount of $\mathrm{CM}$ cases as defined in our study. The 2 farms where the model wrongly as- 
signed as needs attention in multiple quarters of 2013 had higher than average BTM SCC and a higher than average percentage of cattle with an elevated SCC in all quarters of the year. Earlier studies showed a difference between farmers in observing CM based on alterations in milk color and texture (Lam et al., 1993). Possibly, these 2 farmers only observed more severe CM cases in their herd. The farmers that observed high amounts of $\mathrm{CM}$ when the model did not assign as needs attention had, on average, a lower amount of antibiotic supplied to them and lower values for SCC parameters. Some of these herds might have suffered from gram-negative $\mathrm{CM}$ infections that might not be reflected in a high BTM SCC (Barkema et al., 1998). Another possible explanation is that these farmers were very precise in observing and registering $\mathrm{CM}$ and that they assign $\mathrm{CM}$ to even the smallest deviation in udder or milk.

Given the aim of the model, to detect herds with CM problems, it is doubtful whether it is a problem that the model misclassified some herds. The specificity of the presented model was high (96\%) but the sensitivity was fairly low (32\%). Nevertheless, these values are depending on the chosen cut-off values and can be altered depending on the aim of the model and in accordance with the stakeholders (informing farmers, detecting farmers with problems, motivating farmers to improve udder health and decrease CMI, and so on).

The parameters that were included in the best models were selected based on their predictive capacities for CMI and were not necessarily significantly associated with the CMI (could also be included as confounders). Nevertheless, in our model most parameters showed significant associations. In the Results section only the parameters and not the estimates were presented because they could be misinterpreted as explanatory relations. Further, it is not clear whether the parameter estimates are also valid for dairy herds with an automatic milking system and for dairy herds outside the Netherlands. The distribution of the model predictors might differ substantially between dairy herds with a conventional versus an automatic milking system and might also differ between dairy industries in different countries. Therefore, the validity of the predictive model for these herds needs to be further investigated.

For our study, a large group of 1,350 dairy farmers with a conventional milking system was randomly selected and asked to participate. Farms with an automatic milking system were excluded because CM detection in these herds differs substantially from CM detection in herds with a conventional milking system. In the Netherlands, about $15 \%$ of dairy herds use an automatic milking system. The first 240 farmers that responded to the request for participation were included in our study. This might have resulted in some selection bias, because these farmers might have had a higher than average interest in udder health in their herd and, therefore, might not be completely representative for the rest of the population. The study design required a high amount of dedication from the farmers because it involved administrative obligations during a whole year. Therefore, the participating farmers needed to be motivated to cooperate in the study. In comparison with the average Dutch dairy herd with a conventional milking system, the study herds appeared slightly larger. Nevertheless, we think that the slightly biased estimate of the true CMI of all dairy herds did not influence the quality of the models that were developed.

In the Netherlands, our study was the first representative estimate of the CMI since 2009. In that year, farmers observed and registered an average CMI of 28.1 (Lam et al., 2013). Their finding showed a lower CMI compared with the CMI of 32.2 that was found in the present study. An increase in CMI was unexpected because the BTM SCC and the prevalence of elevated SCC cows decreased in the Dutch dairy population between 2008 and 2013. During this period the BTM SCC decreased from, on average, 215,000 cells $/ \mathrm{mL}$ in 2008 to 199,000 cells/mL in 2012, and the prevalence of elevated SCC cows decreased from 22.7 to $19.7 \%$ in the same period (GD, 2013). The focus on udder health in the Dutch dairy industry may have led to increased awareness of farmers for CM detection. In our study we also reduced response bias as much as possible with monthly reminders to submit the recorded cases of CM. Therefore, we believe that the observed CMI is very close to the true CMI in Dutch dairy herds.

The predictive models seemed capable of estimating the CMI for all dairy herds and detecting herds with a high CMI. Observing and registering CM by the farmer remains a crucial source of information in dairy management. Nevertheless, this requires a large amount of discipline and is not always feasible. Periodically monitoring CM based on routine data appears a good alternative to inform farmers about their risk status (i.e., need attention) with regard CMI. Combined with a benchmark of the average CMI for all dairy herds, this may stimulate the farmers to improve their udder health. In addition, the models can be used to monitor CM in the Netherlands on, for example, a yearly basis without having to conduct large scale and expensive field studies. It is, however, advisable to revalidate the developed models after the first few years because currently some major alterations are going on in the dairy industry, such as disappearance of the milk quota system and a reduction in AMU, which might influence the CMI. 


\section{CONCLUSIONS}

This study shows that it is possible to estimate and monitor the average CMI in the dairy population with a model based on routinely collected herd data. It was not possible to exactly predict the CMI for each individual dairy herd. Based on routine herd data however, it was possible to accurately detect herds with a high level of CM.

\section{ACKNOWLEDGMENTS}

This study was financed by the Dutch Dairy Association (NZO, The Hague, the Netherlands) and the Dutch Commodity board for Dairy (PZ, The Hague, the Netherlands). We thank all farmers for their participation and their commitment to this study. Furthermore, we thank the data suppliers RVO (The Hague), CRV (Arnhem), and Qlip (Zutphen) for providing the routine herd data of the study herds.

\section{REFERENCES}

Barkema, H. W., H. A. Deluyker, Y. H. Schukken, and T. J. G. M. Lam. 1999. Quarter-milk somatic cell count at calving and at the first six milkings after calving. Prev. Vet. Med. 38:1-9.

Barkema, H. W., Y. H. Schukken, T. J. G. M. Lam, M. L. Beiboer, H. Wilmink, G. Benedictus, and A. Brand. 1998. Incidence of clinical mastitis in dairy herds grouped in three categories by bulk milk somatic cell counts. J. Dairy Sci. 81:411-419.

Berning, L. M., and G. E. Shook. 1992. Prediction of mastitis using milk somatic cell count, N-acetyl- $\beta$-D-glucosaminidase, and lactose. J. Dairy Sci. 75:1840-1848.

CRV (Dutch Royal Cattle Syndicate). 2001. Definitions of standardized milk production, in Dutch. Report: Bedrijfsstandaardkoe. Accessed May 14, 2015. https://www.crv4all.nl/downloads/fokkerij/ naslagwerken/.

CRV (Dutch Royal Cattle Syndicate). 2011. Definitions of somatic cell Count, in Dutch. Report: Uiergezondheid. Accessed May 14, 2015. https://www.crv4all.nl/downloads/fokkerij/naslagwerken/.

Cui, J. 2007. QIC program and model selection in GEE analyses. Stata J. 7:209-220.

de Haas, Y., H. W. Barkema, Y. H. Schukken, and R. F. Veerkamp. 2005. Associations between somatic cell count patterns and the incidence of clinical mastitis. Prev. Vet. Med. 67:55-68.

de Vries, M., E. A. Bokkers, G. Van Schaik, B. Engel, T. Dijkstra, and I. J. De Boer. 2014. Exploring the value of routinely collected herd data for estimating dairy cattle welfare. J. Dairy Sci. 97:715-730.

Dohoo, I., W. Martin, and H. Stryhn. 2009. Veterinary Epidemiologic Research. Atlantic Veterinary College, Charlottetown, PE, Canada.

GD. 2013. Positive developments in udder health (in Dutch). Accessed Mar. 22, 2015. http://issuu.com/gezondheidsdienstvoordieren/ docs/gd_herkauwer_november_2013/14

Huijps, K., T. J. Lam, and H. Hogeveen. 2008. Costs of mastitis: Facts and perception. J. Dairy Res. 75:113-120.

Jansen, J., G. Van Schaik, R. J. Renes, and T. J. Lam. 2010. The effect of a national mastitis control program on the attitudes, knowledge, and behaviour of farmers in the Netherlands. J. Dairy Sci. 93:5737-5747.

Lago, A., S. M. Godden, R. Bey, P. L. Ruegg, and K. Leslie. 2011. The selective treatment of clinical mastitis based on on-farm culture results: I. Effects on antibiotic use, milk withholding time, and short-term clinical and bacteriological outcomes. J. Dairy Sci. 94:4441-4456.
Lam, T. J., Y. H. Schukken, F. J. Grommers, J. A. Smit, and A. Brand. 1993. Within-herd and between-herd variation in diagnosis of clinical mastitis in cattle. J. Am. Vet. Med. Assoc. 202:938-942.

Lam, T. J. G. M., B. H. P. Van Den Borne, J. Jansen, K. Huijps, J. C. L. Van Veersen, G. Van Schaik, and H. Hogeveen. 2013. Improving bovine udder health: A national control program in the Netherlands. J. Dairy Sci. 96:1301-1311.

Lemeshow, S., D. Teres, J. Klar, J. S. Avrunin, S. H. Gehlbach, and J. Rapoport. 1993. Mortality Probability Models (MPM II) based on an international cohort of intensive care unit patients. JAMA 270:2478-2486.

McGill, D. M., H. A. Mulder, P. C. Thomson, and J. J. Lievaart. 2014. Selecting an appropriate genetic evaluation model for selection in a developing dairy sector. Animal 8:1577-1585.

McParland, S., E. Lewis, E. Kennedy, S. G. Moore, B. McCarthy, M. O'Donovan, S. T. Butler, J. E. Pryce, and D. P. Berry. 2014. Midinfrared spectrometry of milk as a predictor of energy intake and efficiency in lactating dairy cows. J. Dairy Sci. 97:5863-5871.

Olde Riekerink, R. G. M., H. W. Barkema, D. T. Kelton, and D. T. Scholl. 2008. Incidence rate of clinical mastitis on Canadian dairy farms. J. Dairy Sci. 91:1366-1377.

Pan, W. 2001. Akaike's information criterion in generalized estimating equations. Biometrics 57:120-125.

Sampimon, O., B. H. van den Borne, I. Santman-Berends, H. W. Barkema, and T. J. G. M. Lam. 2010. Effect of coagulase-negative staphylococci on somatic cell count in Dutch dairy herds. J. Dairy Res. 77:318-324.

Santman-Berends, I., S. Luttikholt, R. Van den Brom, G. Van Schaik, M. Gonggrijp, H. Hage, and P. Vellema. 2014. Estimation of the use of antibiotics in the small ruminant industry in the Netherlands in 2011 and 2012. PLoS ONE http://dx.doi.org/http:// dx.doi.org/10.1371/journal.pone.0105052.

Santman-Berends, I. M. G. A., R. G. M. Olde Riekerink, O. C. Sampimon, G. van Schaik, and T. J. G. M. Lam. 2012. Incidence of subclinical mastitis in Dutch dairy heifers in the first 100 days in lactation and associated risk factors. J. Dairy Sci. 95:2476-2484.

SDA. 2014. Usage of antibiotic in agricultural livestock in the Netherlands in 2013, in Dutch. Accessed May 15, 2015. http://www. autoriteitdiergeneesmiddelen.nl/en/publications.

Stata. 2014. Data-Analysis and Statistical Software. StataCorp LP College Station, TX.

Steyerberg, E. W. 2009. Clinical Prediction Models: A Practical Approach to Development, Validation, and Updating. Springer Science and Business Media, New York, NY.

Sun, C., P. M. Van Raden, J. B. Cole, and J. R. O'Connell. 2014 Improvement of prediction ability for genomic selection of dairy cattle by including dominance effects. PLoS ONE 9:e103934. http://dx.doi.org/10.1371/journal.pone.0103934.

Thrusfield, M., C. Ortega, I. de Blas, J. P. Noordhuizen, and K. Frankena. 2001. WIN EPISCOPE 2.0: Improved epidemiological software for veterinary medicine. Vet. Rec. 148:567-572.

Trevisi, E., A. Zecconi, S. Cogrossi, E. Razzuoli, P. Grossi, and M. Amadori. 2014. Strategies for reduced antibiotic usage in dairy cattle farms. Res. Vet. Sci. 96:229-233.

van den Borne, B. H. P., G. Van Schaik, T. J. G. M. Lam, and M. Nielen. 2010. Variation in herd level mastitis indicators between primi- and multiparae in Dutch dairy herds. Prev. Vet. Med. 96:49-55.

van den Borne, B. H. P., J. C. Vernooij, A. M. Lupindu, G. Van Schaik, K. Frankena, T. J. Lam, and M. Nielen. 2011. Relationship between somatic cell count status and subsequent clinical mastitis in Dutch dairy cows. Prev. Vet. Med. 102:265-273.

Vergara, C. F., D. Döpfer, N. B. Cook, K. V. Nordlund, J. A. McArt, D. V. Nydam, and G. R. Oetzel. 2014. Risk factors for postpartum problems in dairy cows: Explanatory and predictive modeling. J. Dairy Sci. 97:4127-4140.

Wolff, C., M. Espetvedt, A. K. Lind, S. Rintakoski, A. Egenvall, A. Lindberg, and U. Emanuelson. 2012. Completeness of the disease recording systems for dairy cows in Denmark, Finland, Norway and Sweden with special reference to clinical mastitis. BMC Vet. Res. 8:131. http://dx.doi.org/10.1186/1746-6148-8-131. 
ESTIMATING CLINICAL MASTITIS WITH HERD DATA

6975

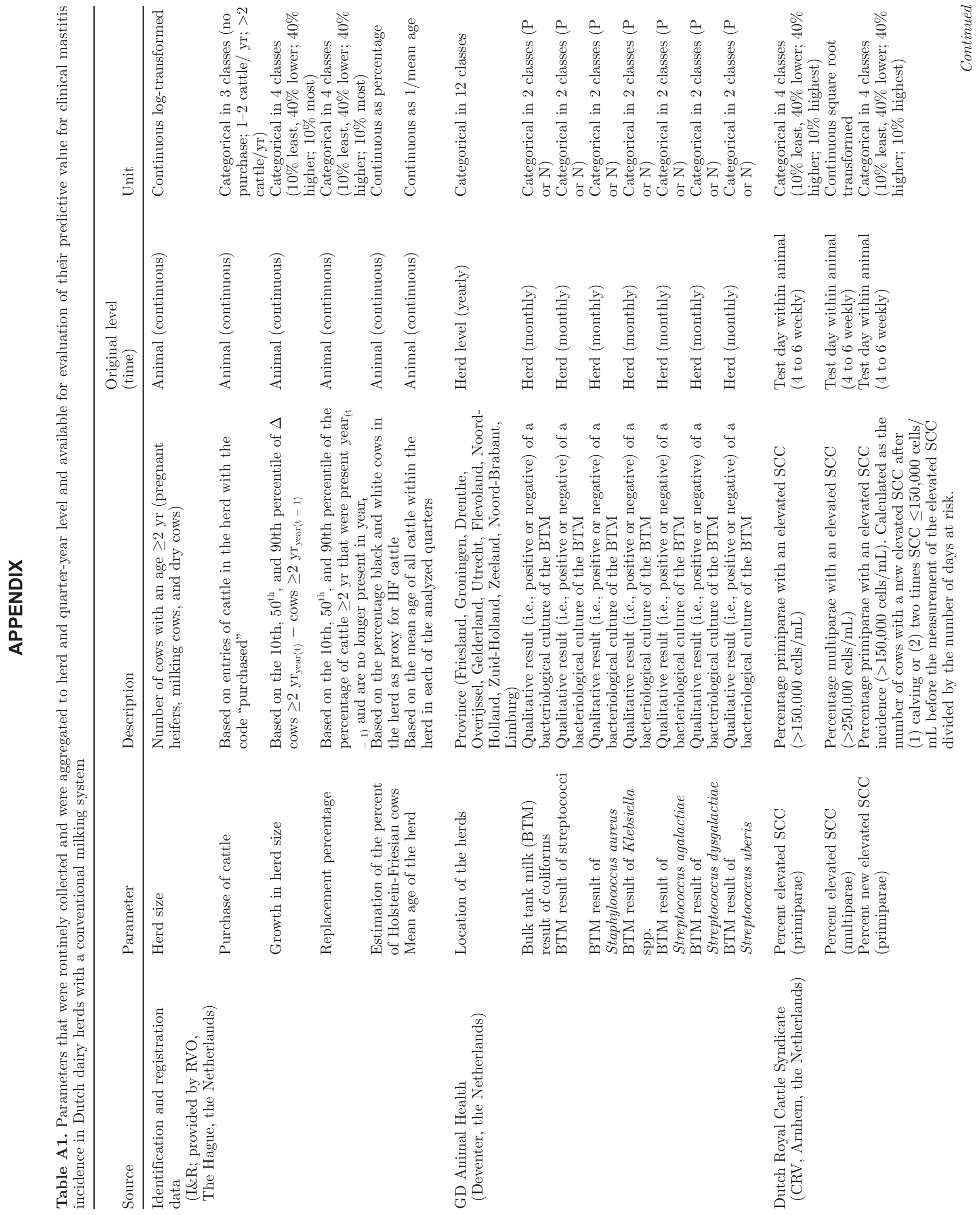




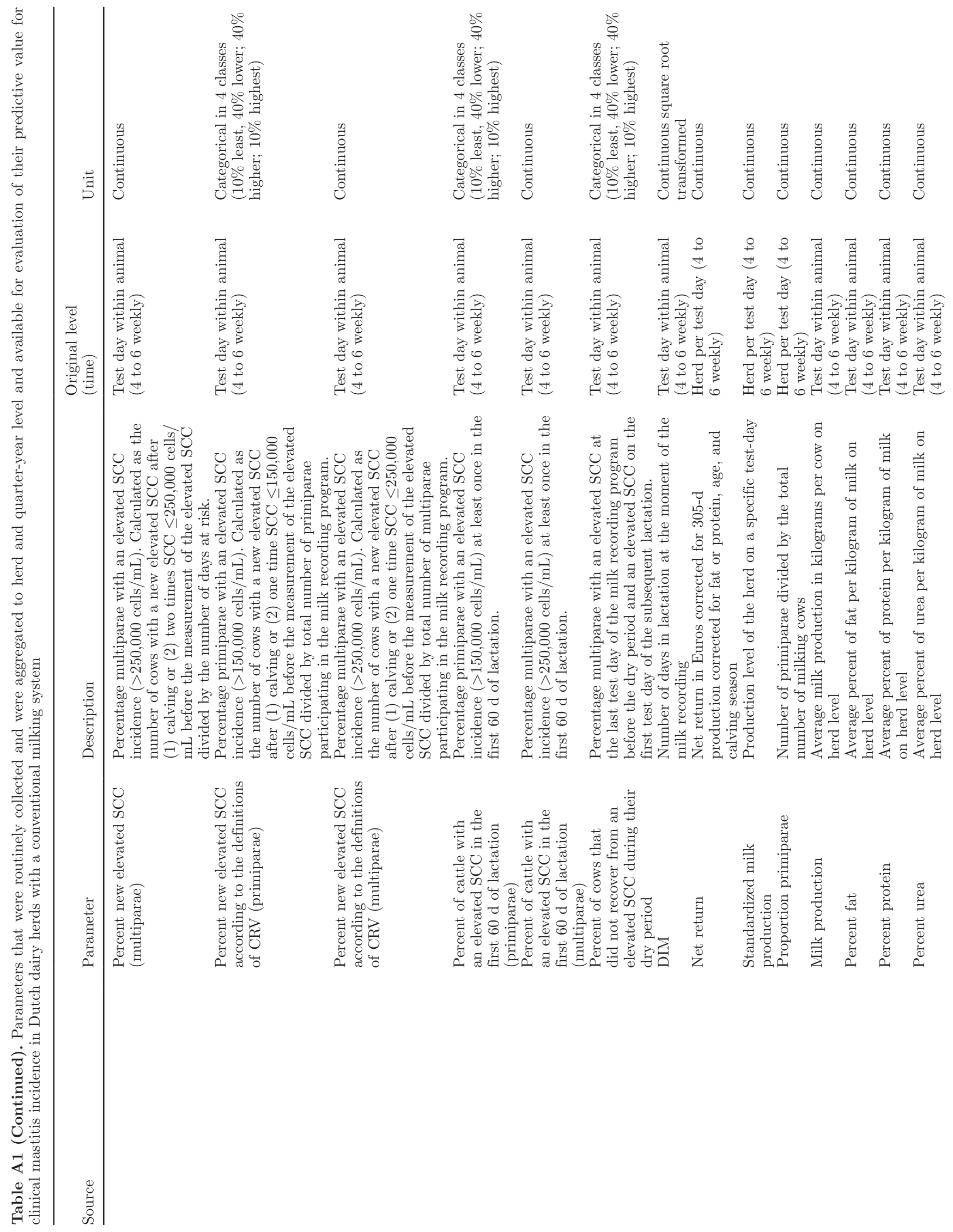


ESTIMATING CLINICAL MASTITIS WITH HERD DATA

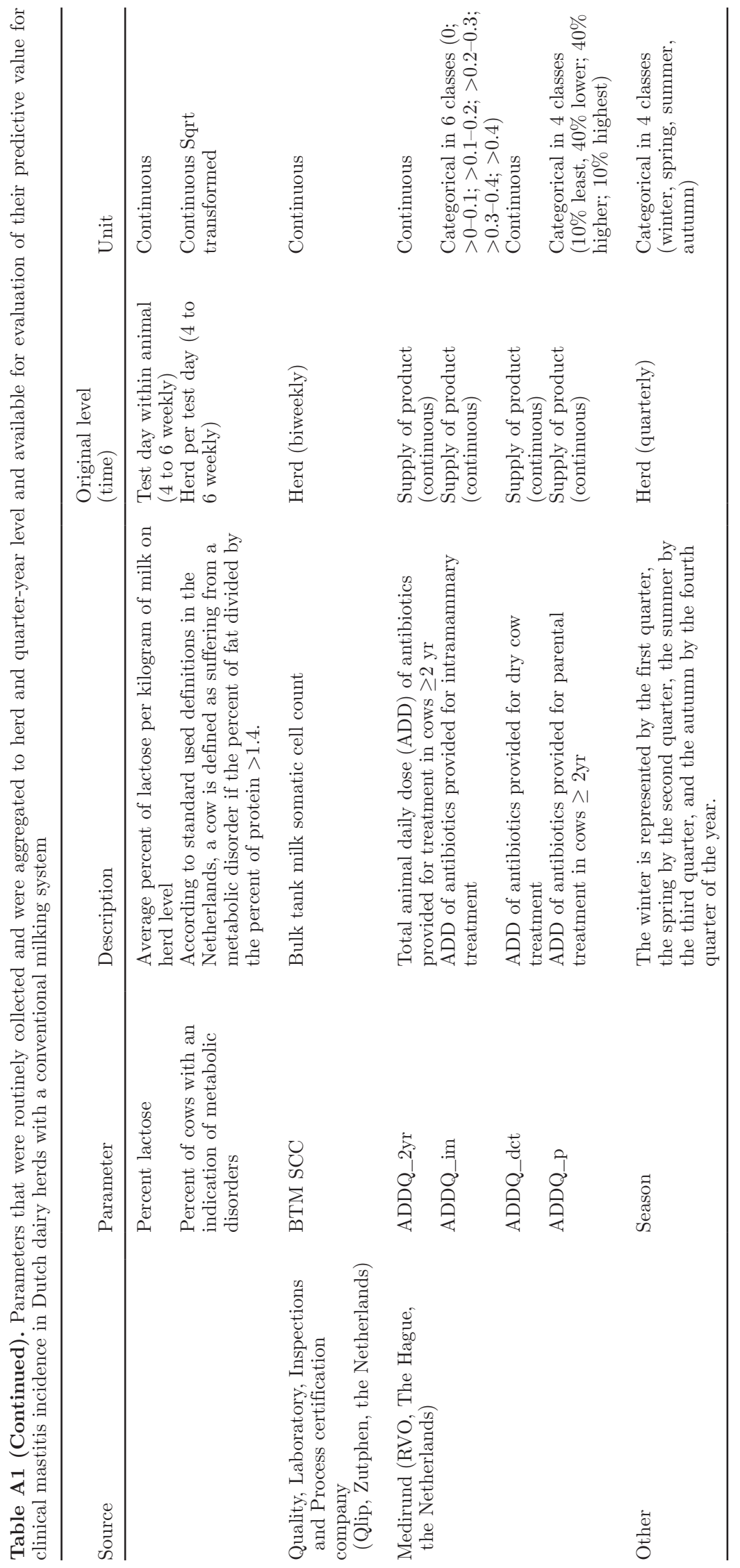

